\title{
Effects of flattening filter (FF) and flattening filter-free (FFF) beams on small-field and large-field dose distribution using the VMAT treatment plan
}

\author{
Dominika J. PlAZAa, Klaudia M. ORZECHOWSKAa, Krzysztof T. ŚLOSAREKa, \\ ${ }^{a}$ Maria Sktodowska - Curie National Research Institute of Oncology, Gliwice Branch, Poland; Radiotherapy Planning \\ Department \\ *E-mail address: Krzysztof.Slosarek@io.gliwice.pl
}

\begin{abstract}
Introduction: The aim of the study was to evaluate the influence of flattening filter (FF) and flattening filter-free (FFF) beams on small-field and large-field dose distribution using the VMAT treatment plan.

Material and methods: Dose distribution calculations were performed for the VMAT technique in two locations: the larynx (small irradiation field; average $30.1 \mathrm{~cm}^{2}$ ) and gynecology (large irradiation field; average $173.1 \mathrm{~cm}^{2}$ ) using X$6 \mathrm{MV}$ flattening filter (FF) and flattening filter-free (FFF) beams. The following values were compared: the number of monitor units, minimum doses, average doses in PTV and maximum average doses in OaR (spinal cord - in larynx radiotherapy, bladder and rectum - in gynecological radiotherapy) and RPI (Radiation Planning Index) coefficient.

Results and Discussion: The performed statistical tests indicate that there is a significant difference $(\mathrm{p}<0.05)$ between the number of monitor units in the irradiation of large (gynecological) fields between the FF and FFF beams. The dose distributions show no statistically significant differences between the flattening filter and flattening-free filter beams (regardless of the field size).

Conclusions: Due to the smaller number of monitor units, it is recommended to use flattening filter beams (FF) for largefield radiotherapy.
\end{abstract}

Key words: Flattening Filter-Free Beams (FFF); Flattening Filter Beams (FF); VMAT (Volumetric Modulated Arc Therapy).

\section{Introduction}

Increased incidence of cancer develops higher demand for radiotherapy which is associated with the improvement of the dynamic irradiation techniques. Radiation therapy can be used as a standalone treatment, or as a treatment combined with surgery, chemotherapy, hormone therapy or immunotherapy. ${ }^{1,2}$

Preparing a treatment plan is a complex process in which the physicist's and doctor's close collaboration plays a key role. The doctor indicates the location of the neoplastic lesion and provides information on the dose (total and fractional) to be administered in the treated area based on therapeutic protocols. It depends on the type of neoplastic lesion, general condition of the patient and guidelines for acceptable doses in healthy tissues. Then, the physicist defines the parameters of the accelerator using Treatment Planning System (TPS) to choose the irradiation technique, energy, geometry of the therapeutic beams and their amount. The experience of a medical physicist allows creating a plan that is most favorable to the patient. ${ }^{3-5}$

When it comes to radiotherapy, until the beginning of the $90 \mathrm{~s}$ the shape of the irradiation field allowed to recreate only the simplest shapes. However, we know perfectly well that the shape of the tumor and the organs surrounding it are irregular. The technical progress made it possible to match the isodose distribution to the shape of the irradiated area thanks to the use of a Multi-Leaf Collimator (MLC). The two opposite collimators have been divided into independently moving leaves, which change their position during the irradiation of the patient, blocking some fractions of the radiation beam. This solution allows for more thorough protection of critical organs. The calculations were made on the True Beam Varian Medical Systems accelerator with a 120-blade MLC HD. While the standard MLC 120 is characterized by the construction of two A and B cassettes, 60 leaves each (the first 10 leaves are $0.5 \mathrm{~cm}$ wide, the next 40 sheets are $0.25 \mathrm{~cm}$ wide, again 10 sheets are $0.5 \mathrm{~cm}$ wide), in the HD version the width of the leaves used is reduced by half. Multi-Leaf Collimator consists of a different number of leaves (of different thickness, which determines different dimensions of the shaped therapeutic field).

In the currently most popular technique using Intensity Modulated Radiation Therapy (IMRT), the shape of the radiation field changes during the irradiation of the patient 
by changing the position of the collimator leaves. The IMRT technique is carried out in the fixed position of the accelerator head. On the other hand, the combination of rotational technique with IMRT is called the Volumetric Modulated Arc Therapy technique (VMAT) and is carried out not only by the defined mobility of the leaves of the multi-leaf collimator, but also by the simultaneous rotation of the accelerator arm with the modulation of the rotation speed and dose rate. This technique is used frequently in very small field radiosurgery and is also increasingly used in standard irradiation techniques. ${ }^{6-8}$

Until now, as far as dynamic techniques are concerned, the standard was the use of flattening filter beams (FF). Currently, medical accelerators allow the use of flattening filter-free photon beams (FFF). Thanks to the appropriate calculation algorithms, the possibility of using them in dynamic techniques significantly increases the possibilities of dose distribution planning. An important factor increasing the interest in using non-planar FFF beams is the use of a higher dose rate, which shortens the irradiation time. Reducing the irradiation time increases the chance that the patient will not move during the treatment session. Changing the position of the patient may result in administrating a lower dose for the treated area, and an increase in the dose for critical organs. This will result in a reduced Tumor Control Probability (TCP) and an increased Normal Tissue Complication Probability (NTCP). ${ }^{9-15}$

FFF beams are often used for stereotaxic techniques. Smaller field size and higher fractional dose makes the choice of filterfree beams very advantageous. ${ }^{2,16}$ The flattening filter produces scattered radiation which increases the dose both on the surface and outside the irradiation field. Therefore, the use of filter-free beams minimizes the occurrence of scattered radiation, which is beneficial in radiotherapy. ${ }^{17-18} \mathrm{~A}$ question may be asked whether the FF and FFF beams can be used alternatively, regardless of the field size. Higher dose rates of the FFF beams shorten the therapeutic session time. That is definitely a big advantage. If they will not worsen the dose distribution, their use would be fully justified. We can use many different coefficients to compare dose distributions in radiotherapy. One of them is Radiation Planning Index (RPI), which allows the compare theoretical and reconstructed treatment plans for one patient. Treatment plans are compared on the basis of the Dose Volume Histogram (DVH) - a graph of dose dependence on the target volume and critical structures. The calculations compare the protection of the critical structures (which are different depending on the location of the tumor) and the radiation of the target. RPI values are in the range $0-1$. Where RPI $=1$, it would be the most advantageous because the OaR's structures would receive no dose and the irradiation volumes would be covered with a homogeneous dose. ${ }^{19}$

The aim of this study was to check whether flattening filter beams and flattening filter-free beams can be used interchangeably regardless of the field dimensions.

\section{Materials and Methods}

The purpose of this study was to compare the calculations of the dose distribution in the VMAT technique for small field radiation - larynx and large field radiation- gynecological radiotherapy using X-6MV flattening filter (FF) and flattening filter-free (FFF) beams. For each patient, calculations were made using a new plan optimization using the same geometry of the beams. The constraints used for optimization were derived from an iterative treatment planning system in beam-FF mode and used for optimization in beam-FFF mode. No additional constraints or adaptations were used for the sake of comparability of plans and to avoid pressure on either method. The quality of the beam planning plans could be further improved by adjusting the optimization constraints accordingly. However, this is beyond the scope of this study. The treatment planning system Eclipse v. 15.6 by Varian Medical System was used. All treatment plans were realized on the accelerator True beam v.2.7 Varian Medical Systems. One of the most important elements of this accelerator's equipment is the highly accurate HD 120 multi-leaf collimator. The highest possible dose rate was used for each plan - for the flattening filter beams it was 600 $\mathrm{MU} / \mathrm{min}$, and for flattening filter-free beams it was 1400 $\mathrm{MU} / \mathrm{min}$.

The first group of small field radiation, which included radiotherapy of the larynx (without lymph nodes) consisted of 30 patients (patients with treatment plans). Treatment plans with the VMAT technique were made for each patient. In each case, the total dose was $51 \mathrm{~Gy}$ and the fractional dose was $3 \mathrm{~Gy}$. In the case of laryngeal cancer, the critical organ was primarily the spinal canal. A new optimization of the plan was performed for each patient and for each energy, using the same number and geometry of the beams (two fields with $0^{\circ}$ and $90^{\circ}$ collimation, and the rotation of the head was in the range of $240^{\circ}-120^{\circ}$ and $120^{\circ}-240^{\circ}$ ).

The second group included 32 patients (patients with treatment plans) who were treated for gynecological tumors. In comparison to the dimensions of the fields used in irradiation of the larynx (without lymph nodes), gynecological tumors are considered large fields. All were treated with a total dose of 50.4 Gy in a fractionated dose of 1.8 Gy. Two treatment plans for different energies were made for each patient. The simultaneously used field collimation $\left(30^{\circ}\right.$ and $\left.330^{\circ}\right)$ and the number of beams (two beams with head rotation $181^{\circ}-179^{\circ}$, $179^{\circ}-181^{\circ}$ ) were identical in each plan. In the case of gynecological tumors, the bladder, rectum, bone heads and intestines are protected. When it comes to analyzing physical dose distributions, the differences in the dose par fraction that exist between the groups do not matter.

The following were compared in the two groups: the number of monitor units, minimum and average doses in PTV and maximum, average doses in OaR spinal cord - in larynx radiotherapy, bladder and rectum - in gynecological radiotherapy and RPI factors. The statistical differences between 
the groups were set at $p=0.05$ for the Mann-Whitney U Test (non-parametric for independent groups).

\section{Results}

Tables 1 and $\mathbf{2}$ show the mean values and standard deviation of the sum of the monitor units, minimum doses in PTV, maximum doses in OaR and mean doses in PTV and OaR.

Figure 1 shows exemplary dose distributions for both analyzed groups; large (gynecological) and small (larynx) fields. The average dimension of the small field is $30.1 \mathrm{~cm}^{2}$, SD -12.4 $\mathrm{cm}^{2}$ and the large $-173.1 \mathrm{~cm}^{2}, \mathrm{SD}-17.6 \mathrm{~cm}^{2}$.
The performed statistical tests indicate that there is no statistically significant difference in the sum of monitor units for small field irradiation between FF and FFF beams (X-6MV). However, for large field radiation, the mean value of units for the FF beam is 613 and for the FFF beam - 742 and these differences are statistically significant $(\mathrm{p}<0.05)$ (Figure 2).

Comparing the dose distributions, in particular the minimum and average doses in PTV and the maximum and average doses in OaR, no statistically significant differences were found between the FF and FFF beams for small and large fields (Table 3).

Table 1. Results of the sum of MU, doses: minimum, maximum and mean values for the FF X-6MV and FFF X-6MV beams for small irradiation fields. OaR1 stands for the spinal cord.

\begin{tabular}{lcccccc}
\hline \hline & \multicolumn{5}{c}{ Larynx, small fields - 30.1 [cm $^{2}$ ] } \\
\hline \multirow{2}{*}{ FF X-6MV } & Sum MU & Dmin_PTV [Gy] & Davg_PTV [Gy] & Dmax_OaR1 [Gy] & Davg_OaR1 [Gy] \\
& SD & 592.6 & 43.3 & 52.3 & 17.9 & 3.5 \\
\multirow{2}{*}{ FFF X-6MV } & 144.1 & 2.6 & 0.3 & 3.2 & 1.5 \\
& Mean & 617.6 & 43.5 & 52.2 & 16.9 & 3.3 \\
\hline \hline
\end{tabular}

Table 2. Results of the sum of MU, doses: minimum, maximum and mean for the FF X-6MV and FFF X-6MV beams for large irradiation fields. OaR1 stands for bladder and OaR2 stands for the rectum.

\begin{tabular}{|c|c|c|c|c|c|c|c|c|}
\hline \multicolumn{9}{|c|}{ Gynecology, large fields - $173.1\left[\mathrm{~cm}^{2}\right]$} \\
\hline & & Sum MU & Dmin_PTV [Gy] & Davg_PTV [Gy] & Dmax_OaR1 [Gy] & Davg_OaR1 [Gy] & Dmax_OaR2 [Gy] & Davg_ OaR2 [Gy] \\
\hline \multirow{2}{*}{ FF X-6MV } & Mean & 613.8 & 47.1 & 51.5 & 52.9 & 29.7 & 51.8 & 23.9 \\
\hline & SD & 81.3 & 1.1 & 0.5 & 1.1 & 2.6 & 1.6 & 3.9 \\
\hline \multirow{2}{*}{ FFF X-6MV } & Mean & 742.7 & 46.9 & 51.5 & 53.1 & 29.6 & 52.1 & 23.6 \\
\hline & SD & 107.5 & 1.3 & 0.7 & 1.0 & 3.0 & 1.4 & 3.8 \\
\hline
\end{tabular}

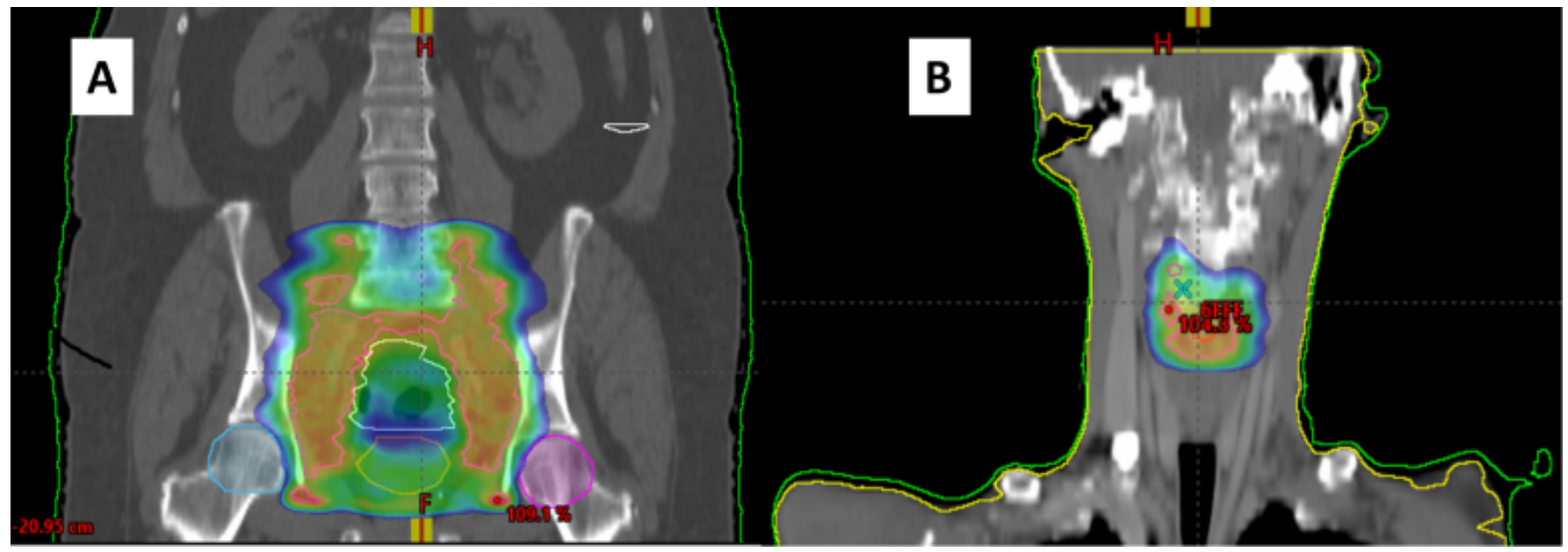

Figure 1. Dose distributions and exemplary dimensions of irradiation fields: A- large fields, gynecological and B - small fields irradiation of the larynx. 

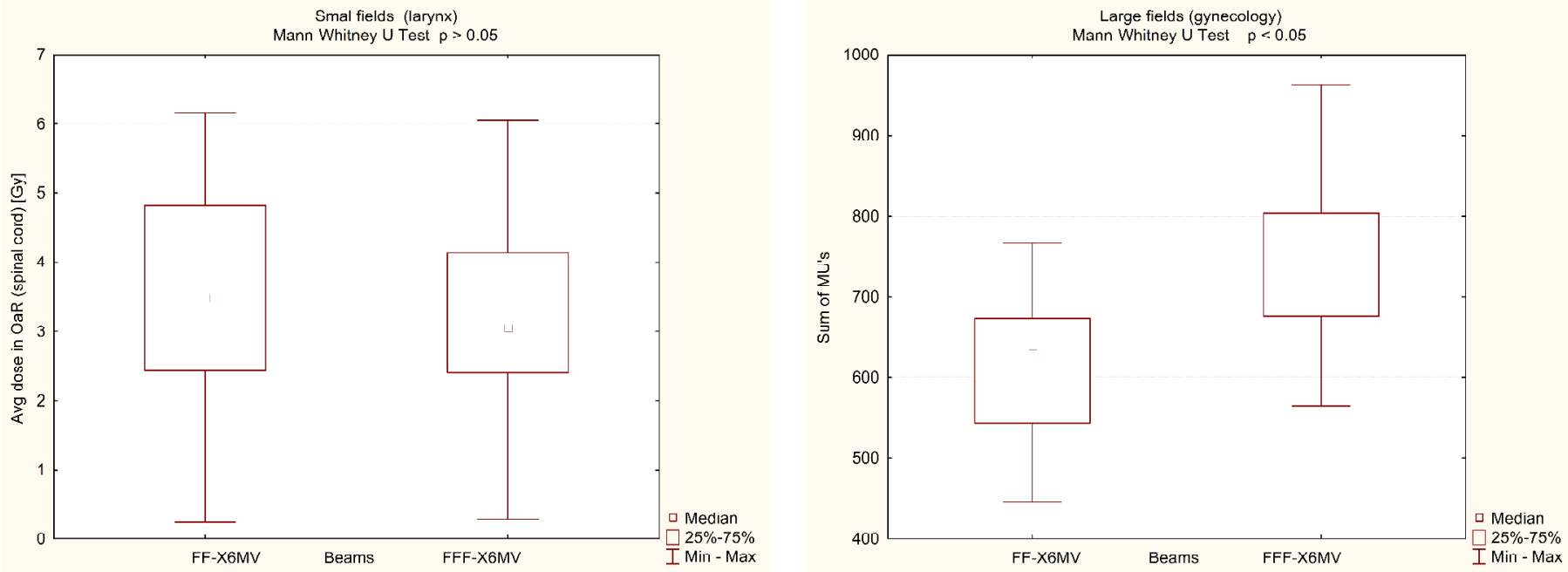

Figure 2. Shows the results of comparing the number of small and large field monitor units for FF and FFF beams.

Table 3. The "p" values of the Mann-Whitney U statistical test assessing the relationship between beams with and without flattening filter for doses in small and large fields.

\begin{tabular}{|c|c|c|c|c|c|c|}
\hline & \multicolumn{3}{|c|}{ Minimum dose [Gy] in PTV } & \multicolumn{3}{|c|}{ Average dose [Gy] in PTV } \\
\hline & FF & FFF & $\mathbf{p}$ & FF & FFF & $\mathbf{p}$ \\
\hline small fields & 43.3 & 43.5 & $>0.05$ & 52.3 & 52.2 & $>0.05$ \\
\hline \multirow[t]{3}{*}{ large fields } & 47.1 & 46.9 & $>0.05$ & 51.5 & 51.5 & $>0.05$ \\
\hline & \multicolumn{3}{|c|}{ Maximum dose [Gy] in OaR1 } & \multicolumn{3}{|c|}{ Average dose [Gy] in OaR1 } \\
\hline & FF & FFF & p & FF & FFF & $\mathbf{p}$ \\
\hline \multirow{2}{*}{ large fields } & \multicolumn{3}{|c|}{ Maximum dose [Gy] in OaR2 } & \multicolumn{3}{|c|}{ Average dose [Gy] in OaR2 } \\
\hline & FF & FFF & $\mathbf{p}$ & FF & FFF & $\mathbf{p}$ \\
\hline small fields & & & $>0.05$ & & & $>0.05$ \\
\hline large fields & 51.8 & 52.1 & $>0.05$ & 23.9 & 23.6 & $>0.05$ \\
\hline
\end{tabular}

\section{Discussion}

For several years, research on flattening-free photon beams of therapeutic devices has been very popular. An extremely important aspect one has to take into consideration while comparing their physical parameters is the appropriate assessment of the treatment plans using this type of energy (you should compare, among others: the number of monitor units, doses received in the target and critical organs).

For large fields (gynecological radiotherapy), no significant statistical differences were found in dose distribution between FF and FFF beams (The Mann-Whitney U test- values are presented in Table 3). The minimum dose for each individual plan, regardless of the treatment and irradiation technique, was about $93 \%$ of the set dose (as indicated in Table 2), while the average dose for the entire target area was over $100 \%$. The number of MU was significantly higher in the FFF beams resulting in a $21 \%$ increase in the number of monitor units compared to the plan on the FF beams. Due to the shape of the dose profile, the dose is lower beyond the central axis for the FFF beams and the additional MU allows the dose to be delivered away from the beam axis. ${ }^{18}$
Therefore, for irradiation of large fields, such as gynecological radiotherapy and breast radiotherapy, it is recommended to use beams with a flattening filter, for the same energy of the radiation beams. Large irradiation fields are used for bigger volumes and greater depths. Thus, it seems we can use higher radiation energies ${ }^{20,21}$ for e.g. gynecology. Higher energy makes the number of monitor units smaller and the dose distributions comparable. In order to test this hypothesis, further research is needed in this area.

Flattening filter-free beams are commonly used for stereotaxic techniques. However, both FF and FFF beams can be used in small field radiotherapy. The calculations performed for 30 patients showed that the minimum point dose for both types of beams was about $85 \%$ of the set dose (as indicated in Table 2), while the mean dose for the entire treated area was $102 \%$ of the set dose. The MU value (mean) only differs by $4 \%$. The use of unfiltered beams in the case of small laryngeal fields also does not increase the benefits in terms of plan quality in accordance with literature values. ${ }^{15}$ As far as dose comparison is concerned, the value of the monitor units and other parameters of the plan do not outweigh the use of one beam instead of the other. 
It seems justified to carry out further research to check what energy is recommended depending on the size of the irradiated area and whether there will be statistically significant differences in the case of other energies. Information on how to define the appropriate energy will allow creating a more favorable treatment plan. This knowledge will be useful not only for physicists, but also for trainees and radiation oncologists.

\section{Conclusions}

While irradiating large and small fields, both FF and FFF beams can be used since dose distributions show no statistically significant differences. However, it is recommended to use flattening-filter beams for large fields due to the smaller number of monitor units.

\section{References}

1. Ślosarek K. Podstawy planowania leczenia w radioterapii. Gliwice: Polskie Towarzystwo Onkologiczne, Oddział Śląski; 2007.

2. Malicki J, Ślosarek K. Planowanie leczenia i dozymetria w radioterapii. Gdańsk: VIA MEDICA; 2016.

3. Waligórski M, Lesiak J. Podstawy Radioterapii. Warszawa: PWN; 2000.

4. $\quad$ Łobodziec W. Dozymetria promieniowania jonizującego w radioterapii. Katowice: Wyd. UŚ; 1999.

5. Kukołowicz P. Charakterystyka wiązek terapeutycznych fotonów i elektronów. Kielce: RTA; 2001.

6. Van Dam J, Marinello G. Methods for in vivo dosimetry in external radiotherapy. Brussels: ESTRO; 1994.

7. Jia F, Xu D, Yue H, Wu H, Li G. Comparison of Flattening Filter and Flattening Filter-Free Volumetric Modulated Arc Radiotherapy in Patients with Locally Advanced Nasopharyngeal Carcinoma. Med Sci Monit. 2018;24:8500-8505. https://doi.org/10.12659/MSM.910218

8. Ma C, Chen M, Long T, Parsons D, et al. Flattening filter free in intensity modulated radiotherapy (IMRT) - Theoretical modeling with delivery efficiency analysis. Med Phys. 2019;46(1):34-44. https://doi.org/10.1002/mp.13267

9. Alvarez Moret J, Obermeier T, Pohl F. Second cancer risk after radiation therapy of ependymoma using the flattening filter free irradiation mode of a linear accelerator. J Appl Clin Med Phys. 2018;19(5):632-639. https://doi.org/10.1002/acm2.12438

10. Wang L, Ding G. Estimating the uncertainty of calculated out-of-field organ dose from a commercial treatment planning system. J Appl Clin Med Phys. 2018;19(4):319-324. https://doi.org/10.1002/acm2.12367

11. Yao C, Chang T, Lin C. Three-dimensional dose comparison of flattening filter (FF) and flattening filter-free (FFF) radiation therapy by using NIPAM gel dosimetry. PLoSOne. 2019;14(2):e0212546. https://doi.org/10.1371/journal.pone.0212546

12. Irazola L, Sánchez-Nieto B, García-Hernández M. 10-Mv SBRT FFF Irradiation Technique is associated to the lowest peripheral dose: the outcome of 142 treatment plans for the 10 most common tumor locations. Radiat Prot Dosimetry. 2019;185(2):183-195. https://doi.org/10.1093/rpd/ncy292

13. Aoki S, Yamashita H, Haga A. Flattening filter-free technique in volumetric modulated arc therapy for lung stereotactic body radiotherapy: A clinical comparison with the flattening filter technique. Oncol Lett. 2018;15(3):3928-3936. https://doi.org/10.3892/ol.2018.7809

14. Duane S. Dosimetry for Flattening Filter Free (FFF) linac beams and small fields (SF). National Physics Laboratory, 2013.

15. Dobler B, Obermeier T, Hautmann M. Simultaneous integrated boost therapy of carcinoma of the hypopharynx/larynx with and without flattening filter - a treatment planning and dosimetry study. Radiat Oncol. 2017;12(1):114. https://doi.org/10.1186/s13014-017-08508

16. Maier J, Knott B, Maerz M. Simultaneous integrated boost (SIB) radiation therapy of right sided breast cancer with and without flattening filter - A treatment planning study. Radiat Oncol. 2016;11(1):111. https://doi.org/10.1186/s13014-016-0687-6

17. Dobler B, Maier J, Knott B. Second Cancer Risk after simultaneous integrated boost radiation therapy of right sided breast cancer with and without flattening filter. Strahlenther Onkol. 2016;192(10):687-95. https://doi.org/10.1007/s00066-016-1025-5

18. Baic B, Kozłowska B, Kwiatkowski R, Dybek M. Clinical advantages of using unfl attened 6-MV and 10-MV photon beams generated by the medical accelerator Elekta Versa HD based on their dosimetric parameters in comparison to conventional beams. Nukleonika;2019:64(3):77-86. https://doi.org/10.2478/nuka-2019-0010

19. Ślosarek K, Grządziel A, Szlag M, Bystrzycka J. Radiation Planning Index for dose distribution evaluation in stereotactic radiotherapy. Reports of Practical Oncology and Radiotherapy. 2008;13(4):182-186. https://doi.org/10.1016/S1507-1367(10)60007-7

20. Leszczyński W, Ślosarek K, Szlag M. Comparison of dose distribution in IMRT and RapidArc technique in prostate radiotherapy.Reports of Practical Oncology and Radiotherapy, 2012;17(6):348-351. https://doi.org/10.1016/j.rpor.2012.05.002

21. Radwan M, Grządziel A, Hawrylewicz L, Ślosarek K, Osewski W. The influence of photon energy on dose distribution for IMRT and VMAT plans. Nowotwory Journal of Oncology. 2014;64(3):230-236. https://doi.org/10.5603/NJO.2014.0037 